Treviño Villarreal, D. C. y González Medina, M. A. (2020). Involucramiento docente y condiciones del aula: una díada para mejorar la convivencia escolar en bachillerato. Revista de Investigación Educativa, 38(2), 397-414.

DOI: http://dx.doi.org/10.6018/rie.372241

\title{
Involucramiento docente y condiciones del aula: una díada para mejorar la convivencia escolar en bachillerato
}

\section{Teacher involvement and classroom conditions: a dyad to improve school coexistence in baccalaureate}

\author{
Diana Carolina Treviño Villarreal* y Mario Alberto González Medina** \\ * Secretaría de Educación del estado de Nuevo León (México) \\ **Universidad de Monterrey (México)
}

\begin{abstract}
Resumen
Una mejora en la convivencia escolar resulta ser un baluarte que viene a influir en las relaciones que se dan al interior de las escuelas, no obstante, para alcanzar dicha mejora, es necesario conocer aquellos elementos que la favorecen. Derivado de esto, el objetivo del presente trabajo es determinar la relación entre los factores Atención, Participación, Sanciones y Condiciones del Aula con la Convivencia escolar en alumnos de bachillerato. La muestra estuvo conformada por 47397 studiantes de la República Mexicana; fue utilizado un cuestionario que forma parte de la Evaluación de Condiciones Básicas para la Enseñanza y el Aprendizaje (ECEA) realizado por el Instituto Nacional para la Evaluación en México. Después de una revisión de la literatura, se propuso un modelo teórico con los factores mencionados. Posteriormente, fue aplicada la técnica de modelaje con ecuaciones estructurales y se obtuvo un modelo empírico. Se hizo evidente la relación entre los factores citados con la convivencia escolar, esto es, la atención que proporcionan los maestros, la participación que promueven, las sanciones que aplican y las condiciones del aula son cruciales para mejorar la convivencia. Se concluye que tanto el involucramiento de los docentes como de las autoridades educativas es transcendental en este tema.

Palabras clave: educación para la paz; enseñanza secundaria; profesión docente; aula.
\end{abstract}

Correspondencia: Mario Alberto González Medina, marioalberto.gonzalezm@udem.edu, Morones Prieto, 4500 Pte. Colonia Jesús M. Garza, San Pedro Garza García, Nuevo León, México. 


\begin{abstract}
An improvement in school coexistence figures as a decisive factor influencing relationships within the school community. However, in order to achieve such an improvement, it is necessary to know the elements that may favor the aforementioned interaction and community life. Hence, the objective of the present work is to determine the relationship between several factors, namely Attention, Participation, Sanctions and Classroom Conditions, in secondary education. The sample consisted of 47397 students from the Mexican Republic, and a questionnaire conducted by the National Institute for Evaluation in Mexico as part of the Assessment of Basic Conditions for Teaching and Learning (ECEA) was used. After a review of the literature, a theoretical model considering the mentioned factors was proposed. Subsequently, the modeling technique with structural equations was applied and an empirical model was obtained. Here, the relationship between the aforementioned factors and school interaction became evident; that is, the attention provided by the teachers, the participation promoted, the sanctions applied and the conditions of the classroom were proven crucial to improving the school environment. It is hence concluded that both the involvement of teachers and educational authorities are transcendental in this area.
\end{abstract}

Keywords: peace education; secondary education; teaching profession; classroom.

\title{
Introducción
}

Sin duda, el tema de la convivencia ha tenido relevancia a nivel mundial (López, 2014), de ahí la importancia que esta sea favorecida por los países y desde la educación (Secretaría de Educación Pública [SEP], 2016), contexto en el cual debe fundarse en la tolerancia para aceptar las diferentes opiniones de las personas, en el aprecio hacia ellas y en el respeto a su dignidad (Contreras, 2018; Secretaría de Educación Pública del estado de Puebla, 2016). Ahora bien, debido a que, en México, la política educativa ha hecho notorio el valor de su promoción en las escuelas (Instituto Nacional para la Evaluación de la Educación [INEE], 2016a), las acciones de los docentes, la comunidad educativa y las autoridades resultan ser esenciales para que se vayan creando ambientes y condiciones en las aulas que permitan a los estudiantes adquirir aprendizajes vinculados con el desarrollo de habilidades para vivir de forma pacífica e incluyente (SEP, 2016).

\section{Algunas precisiones de la convivencia}

La convivencia es entendida como la capacidad que tienen las personas para vivir con otras (Arredondo Reyes, 2019; Ministerio de Educación, s.f.) y, en un sentido amplio, como la edificación de una paz perdurable (Fierro Evans \& Carbajal Padilla, 2019) que debe estar sustentada en el diálogo y tener principios democráticos, respetando siempre las diferencias. Aprender a convivir es un ejercicio elemental que no anula la presencia de conflictos (Landeros \& Chávez, 2015), ya que estos forman parte de la naturaleza de las personas (Alegría Rivas, 2016; Dopico Rodríguez, 2011; Pérez Archundia \& Gutiérrez Méndez, 2016) pero que debe permitir relaciones de calidad (Landeros \& Chávez, 2015). 
En la escuela, los alumnos aprenden a convivir entre ellos y con los adultos (Centro de Estudio y Acción Social Panameño, 2012), y es precisamente en este espacio en donde acontecen hechos que se constituyen como elementos pedagógicos de gran alcance y que convierten a los maestros en agentes de cambio y transformación (Alvarado Juárez, 2019; Arias Vinasco, 2018; Arredondo Reyes, 2019; Cruz Hernández \& Reyes Ruiz, 2016; Penalva López, 2018), por tanto, la convivencia y las relaciones en las aulas son inseparables de las estrategias metodológicas (Pineda Alfonso \& García Pérez, 2016).

Así pues, debido a que el estudio de la convivencia escolar implica un profundo análisis y la consideración de su interrelación con diferentes factores, destacando aquello que sobreviene al interior de los planteles (Chavez Romo, Gómez Nashiky, Ochoa Cervantes \& Zurita Rivera, 2016; Díaz Better \& Sime Poma, 2016), a continuación, serán abordadas algunas de estas relaciones con base en un riguroso sustento teórico.

\section{Involucramiento docente en el aula: atención, fomento de la participación y sancio- nes; tres factores conexos}

La práctica educativa es una acción reflexiva y ágil (García Cabrero, Loredo Enríquez \& Carranza Peña, 2008), por ello, la educación no debe limitarse solo a la transmisión de conocimientos, sino que debe dar también atención a la formación integral del estudiante (Hernández Prados \& Sánchez Romero, s.f.), por tanto, a los docentes les compete establecer las dinámicas de las relaciones en el aula, aquellas que permitirán crear ambientes enriquecedores y de participación. Asimismo, para que se creen espacios adecuados, resulta fundamental que se establezcan reglas y sanciones (Cámere, 2009). En otras palabras, son valiosas la implementación de prácticas y medidas concretas (Caballero Grande, 2010).

\section{Atención del docente hacia el alumno y su influencia sobre la convivencia escolar}

En palabras de algunos autores, ciertos involucrados en el ámbito de la investigación han expresado ideales relacionados con la convivencia escolar, los cuales apuntan hacia la comunicación asertiva, el compromiso, el trabajo en equipo, entre otros (Peña Figueroa, Sánchez Prada, Ramírez Sánchez \& Menjura Escobar, 2017).

Al respecto, desde la escuela se ha observado que el establecimiento de diversas dinámicas por parte de los maestros disminuye los actos de violencia y generan un buen clima de convivencia (Arón, Milicic, Sánchez \& Subercaseaux, 2017; González Medina \& Treviño Villarreal, 2018). En este sentido, cierto tipo de atención que brindan los docentes a sus estudiantes forja cuestiones positivas en ellos, tal como lo hizo notar Parra (2014), quien manifestó la importancia de que los maestros fueran mediadores en los procesos de enseñanza y aprendizaje, una acción que facilitaría las soluciones en la práctica, a través de sugerencias, persuasión y razonamiento. Todo ello incidiría en las buenas relaciones en el aula.

Concretamente, en bachillerato, la interacción durante el quehacer pedagógico es un elemento que debe ser valorado por los maestros, ya que permite alcanzar 
mejores ambientes y relaciones en el salón de clases. No obstante, para que dicha interacción sea potenciada, el docente debe proporcionar comunicación, respeto y confianza a sus alumnos, algo que facilitará el acercamiento para el diálogo y la consulta de dudas durante las clases (Castañeda Millán, 2014).

En otras palabras, los maestros juegan un papel trascendental en la formación de los alumnos para una convivencia pacífica en la escuela (Secretaría de Educación del Gobierno del estado de México, 2013), por tanto, son ellos quienes deben reflexionar sobre los ajustes por hacer en su labor, un acto que generará un mejor contacto con el alumnado, además, se conseguirán relaciones más afectuosas y de comprensión, y, por ende, dichos sentimientos forjarán en el aula una sana convivencia (Artavia Granados, 2005), no obstante, para alcanzarlo, es indispensable su formación, esto bajo cierto tipo de capacitación en modelación de prácticas pedagógicas para el impulso de ambientes de convivencia basados en la participación y el aprendizaje colaborativo (Reyes Angona, Gudiño \& Fernández Cárdenas, 2018).

\section{Participación de los estudiantes en el aula y su relación con la convivencia escolar}

Acerca de la participación de los alumnos promovida por los docentes durante las clases, se ha manifestado que es trascendental crear en el aula un clima propicio en el que sea posible dicha participación, esto es, que el docente haga propuestas educativas basadas en la relación mutua, lo cual forjaría un proceso de comunicación bidireccional y favorecería los niveles de convivencia (Departamento de Educación, Cultura y Deporte, 2007). Aunado a esto, también se ha discutido que una educación para la convivencia debiera asumir un análisis al currículo, en el cual sería imprescindible que sobresalieran acciones como la participación, la comunicación y el buen trato (Vidanes Díez, 2007).

Al respecto, Murillo Pacheco (2010) señaló criterios para seleccionar actividades de aprendizaje, más concretamente puntualiza que se deben propiciar las interacciones y la participación de los alumnos en el aula; un ejercicio que permitiría la confrontación sobre las diferentes formas de pensar ante cierta situación, y algo que, por consiguiente, les enseñaría a ser tolerantes, reconocer errores y aprender a escuchar; y así se podría dar paso a una mejora en la convivencia. En cuanto a este tema se ha llegado a expresar que los maestros deberían ser intermediarios durante su práctica de una manera más comprometida, en donde lo esencial sea que en cada ejercicio propuesto se cuente con la participación y contribución de todos los integrantes, esto a pesar de que las necesidades de los escolares pudieran ser muchas y fuera difícil solucionarlas en un mismo momento. Por ello, los docentes debieran priorizar las necesidades, ya que con esto se estaría fomentando un desarrollo reflexivo en los alumnos bajo una convivencia armónica (Escobar Medina, 2015; García, Olvera \& Flores, 2006).

Por otro lado, Artavia Granados (2005) llevó a cabo un estudio referente a las interacciones entre alumnos y maestros en el proceso de enseñanza y aprendizaje, y fue utilizado el término "tacto pedagógico", para hacer referencia al tipo de relaciones personales que se dan entre los involucrados en el aula, y donde se incluye el nivel de participación fomentado. La conclusión sería que el tacto pedagógico permite el establecimiento de relaciones empáticas que coadyuvan a la generación de ambientes escolares positivos. 
En suma, la participación es un aspecto predominante que favorece la sana convivencia (López de Mesa Melo, Carvajal Castillo, Soto Godoy \& Urrea Roa, 2013; Muñoz Quezada, Lucero Moncada, Cornejo Araya, Muñoz Molina \& Araya Sarabia, 2014; Red de Apoyo a la Gestión Educativa, 2014; Sandoval Manríquez, 2014), por tanto, esta debiera estar incorporada en el proyecto educativo de todo plantel (Muñoz Quezada et al., 2014).

\section{Las sanciones y su correspondencia con la convivencia escolar}

En virtud de que las personas requieren ejercer a diario una adecuada convivencia (Luna Rivera, 2015), en el contexto educativo se pueden llevar a cabo tareas, como dar atención a las conductas de riesgo y ejercer sanciones dirigidas a subsanar daños y a reintegrar a quienes vulneran las normas, con la finalidad de que dicha convivencia sea más provechosa (Secretaría de Educación Pública del estado de Puebla, 2016). De acuerdo con Landeros y Chávez (2015) el criterio correctivo y autoritario viene a ser un recurso en materia de convivencia, ya que tiene alcance en el reconocimiento de los límites; asimismo, para Colom et al. (2010), una de las respuestas a los problemas de convivencia en la escuela implica mantener acciones, entre ellas, el propiciar una total coordinación con la familia, citando frecuentemente a los padres y favoreciendo el diálogo.

En torno al tema, en ciertas investigaciones se ha señalado que los reglamentos escolares (en los cuales están inmersas las medidas disciplinarias o sanciones) pudieran llegar a ser una herramienta de formación para educar en la convivencia (Ochoa Cervantes \& Diez Martínez, 2013). Además, se ha afirmado que los docentes deberían focalizar su atención en las normas de disciplina, ya que con esto se estaría coadyuvando a conseguir un buen clima escolar (Barreda Gómez, 2012).

Concretamente, en el Reglamento de disciplina escolar de las escuelas de educación públicas y particulares de Educación Básica y Media Superior del estado de Nuevo León, se han dado a conocer medidas como el diálogo entre alumnos, padres y docentes sobre las faltas cometidas, así como los exhortos por escrito (Gobierno del estado de Nuevo León, 2016).

Con esto, resulta sensato suponer que cuando las escuelas poseen métodos de disciplina constante, se aplican sanciones racionales y se afrontan los asuntos que alteran el orden de forma directa y reflexiva, se está favoreciendo la convivencia armónica (Ruiz Cuéllar, s.f.).

\section{Condiciones del aula y convivencia escolar}

El aula es el área institucional favorable para construir la convivencia en la escuela porque es el primer lugar de vida pública de las personas donde se construyen las relaciones sociales (Ianni, 2003). Asimismo, el significado y el grado en que esta se ajusta a las necesidades de las personas involucradas, van a determinar las relaciones con lo que les rodea (Departamento de Educación, Cultura y Deporte, 2007). Al respecto, el Instituto Nacional de la Infraestructura Física Educativa (INIFED, 2013) en México, expresó que cada aula debería proporcionar un ambiente en donde se genere 
la comunicación y las interacciones, además donde la temperatura y la iluminación adecuada son elementales, tal como afirma la SEP (s.f.a) el aula debe ser un lugar bien ventilado e iluminado.

No obstante, recientemente fue dado a conocer que, en México, muchas escuelas presentan condiciones precarias en su infraestructura (Miranda López, 2018). De hecho, en países de América Latina, las condiciones de las aulas no son las apropiadas en cuanto a los sistemas de temperatura e iluminación, solo por mencionar algunas básicas (Bos, Ganimian \& Vegas, 2014).

Ahora bien, la Oficina Regional de Educación para América Latina y el Caribe (OREALC/UNESCO Santiago, 2017) expuso que, dentro de los desafíos de ciertos países, se encuentran el ofrecer suficientes espacios físicos en las escuelas y garantizar que estos se transformen en verdaderas áreas para el aprendizaje.

Por añadidura, Castro Pérez y Morales Ramírez (2015) señalan que tanto el ambiente escolar como el del aula, resultan fundamentales para los niños y maestros, ya que la escuela constituye el espacio en donde pasan un tiempo considerable, por tanto, y de haber necesidades y carencias físicas, como la iluminación y ventilación, se estarían presentando repercusiones en las relaciones entre ellos y en la calidad de los aprendizajes. Rodríguez Vite (s.f.) también abordó el tema, y aseveró que para alcanzar el éxito en el aula es necesario que haya suficiente iluminación y ventilación para el progreso armónico de las actividades.

En este punto es preciso mencionar que las condiciones del aula son un factor que influye en la indisciplina escolar como tal (y por consiguiente en las sanciones que se ejercen). Por tanto, el alumnado debe gozar de comodidad en lo que respecta a espacios, ventilación y luz (Morales Rivas, Marina Narváez \& Sarria Membreño, 2014).

La propuesta de Quintero Corzo, Munévar Molina y Munévar Quintero (2015) habla sobre la importancia de incluir en los planes de desarrollo, estrategias para mejorar los planteles educativos en cuanto a los aspectos arriba mencionados, esto es, ventilación, iluminación, entre otros, con la finalidad de regular las interacciones y mejorar así la calidad de todos los métodos de formación.

En síntesis, el involucramiento docente, en el cual está inmersa la atención, la participación y las sanciones, así como también las condiciones del aula, resultan por demás valiosas en los procesos escolares, y parecieran favorecer una mejor convivencia.

Por consiguiente, el objetivo de este artículo es comprobar dichas relaciones, teniendo en cuenta que la Convivencia escolar hace referencia a ciertas formas de interacción que se dan entre los integrantes de la comunidad educativa y que se presentan en algunos procesos escolares (INEE, 2018); la atención se contempla como el proceso cognitivo que permite enfocar recursos (SEP, s.f.b), y para lo cual se considerará el involucramiento del docente en algunas tareas; la participación se entiende como el poder que tienen las personas para involucrarse en diversos contextos (Pérez Galván \& Ochoa Cervantes, 2017), en este caso, los alumnos en el salón de clases, y que se promueve de manera anticipada por el profesor; las sanciones, direccionadas al castigo frente a una trasgresión, son las impuestas por la autoridad escolar $\mathrm{y}$, por último, las condiciones del aula se definen como el espacio que se encuentra construido en el interior de la escuela (SEP, 2008), concretamente el ambiente físico 
apropiado para el desarrollo de actividades, esto en lo correspondiente, entre otras cosas, a ventilación, temperatura e iluminación (INEE, 2016b).

\section{Método}

\section{Objetivo}

Determinar la relación entre los factores Atención, Participación, Sanciones y Condiciones del aula con la Convivencia escolar en alumnos de Bachillerato de la República Mexicana.

\section{Población y Muestra}

La población estuvo conformada por 4916620 estudiantes que cursaban el último grado de bachillerato en el ciclo escolar 2015-2016 en los 19359 planteles de la República Mexicana (SEP, 2018). La cantidad de alumnas y alumnos fue de 2460355 (50.04\%) y 2.456.265 (49.96\%), respectivamente.

En la muestra se estudiaron 47397 alumnos, con una edad media de 17.1 años y una desviación estándar de .5 años; 24462 (51.6\%) fueron mujeres, 22653 (47.8\%) hombres y $282(.6 \%)$ datos perdidos. El total de estudiantes de planteles públicos fue de 42650 (90\%) y $4747(10 \%)$ de privados. El procedimiento de selección fue probabilístico, estratificado y por conglomerado: se eligieron al azar escuelas autónomas, privadas, estatales y federales y, a la postre, se seleccionaron todos los estudiantes de algunas escuelas. El margen de error y la confianza para el cálculo del tamaño de muestra fueron del 5\% y 95\%, respectivamente.

\section{Instrumento}

El cuestionario utilizado forma parte de la Evaluación de Condiciones Básicas para la Enseñanza y el Aprendizaje en Bachillerato que realizó el Instituto Nacional para la Evaluación de la Educación (INEE) de México. Se utilizaron las bases de datos públicas del INEE que contienen los resultados de los alumnos. En la presente investigación se emplearon solo cinco factores y 16 variables (Tabla 1) del instrumento, los cuales provienen de una búsqueda exhaustiva de la literatura relacionada con la propuesta del modelo teórico (Figura 1). Para asegurar que los factores se midieran a través de las variables propuestas, se realizó la validación del constructo a través de un Análisis Factorial Confirmatorio (AFC) (Figura 2). Con este análisis se obtuvo una $\chi^{2} /$ g.l. $=1.88, \mathrm{p}$-valor $=.000, \mathrm{CFI}=.92, \mathrm{IFI}=.90, \mathrm{TLI}=.96, \mathrm{RMSEA}=.039$. Los resultados del AFC indican que fue válida la medición de cada factor a través de las variables planteadas. Además, la fiabilidad se midió con el coeficiente alfa $(\alpha)$ de Cronbach resultando para cada factor los siguientes valores: Convivencia escolar $(\alpha=$ .792), Atención $(\alpha=.831)$, Participación $(\alpha=.860)$, Sanciones $(\alpha=.822)$ y Condiciones del aula $(\alpha=.901)$. 
Tabla 1

Factores, variables y valores de la variable

\begin{tabular}{|c|c|c|}
\hline Factor & Variable (Pregunta) & $\begin{array}{c}\text { Valores de la } \\
\text { variable } \\
\end{array}$ \\
\hline $\begin{array}{c}\text { Convivencia } \\
\text { escolar }\end{array}$ & $\begin{array}{l}\text { ¿Con qué frecuencia me llevo bien con: } \\
\text { 1. Compañeros? } \\
\text { 2. Hombres y mujeres? } \\
\text { ¿Con qué frecuencia: } \\
\text { 3. Puedo confiar en mis profesores? } \\
\text { 4. Mis profesores aceptan mi forma de ser? } \\
\text { 5. Mis profesores son amables? }\end{array}$ & \\
\hline Atención & $\begin{array}{l}\text { ¿Con qué frecuencia tus profesores? } \\
\text { 1. Resuelven nuestras dudas en la clase cuando } \\
\text { preguntamos } \\
\text { 2. Están atentos al trabajo que realizamos en clase } \\
\text { 3. Nos hacen comentarios o sugerencias sobre las tareas y } \\
\text { los trabajos para que mejoremos }\end{array}$ & $\begin{array}{l}\text { Siempre } \\
\text { Muchas veces } \\
\text { Pocas veces } \\
\text { Nunca }\end{array}$ \\
\hline Participación & $\begin{array}{l}\text { ¿Con qué frecuencia los profesores? } \\
\text { 1. Nos dejan participar en sus clases } \\
\text { 2. Organizan sus clases para que todos los estudiantes } \\
\text { participemos }\end{array}$ & \\
\hline Sanciones & $\begin{array}{l}\text { ¿Con qué frecuencia se aplican las siguientes sanciones } \\
\text { para los estudiantes? } \\
\text { 1. Amonestación verbal } \\
\text { 2. Amonestación escrita } \\
\text { 3. Citatorio a los padres }\end{array}$ & \\
\hline $\begin{array}{c}\text { Condiciones } \\
\text { del aula }\end{array}$ & $\begin{array}{l}\text { ¿En cuántas de las aulas donde tomas clase es adecuada: } \\
\text { La ventilación? } \\
\text { 1. La temperatura? } \\
\text { 2. La iluminación? }\end{array}$ & $\begin{array}{l}\text { Todas } \\
\text { La mitad o más } \\
\text { Menos de la } \\
\text { mitad } \\
\text { Ninguna }\end{array}$ \\
\hline
\end{tabular}




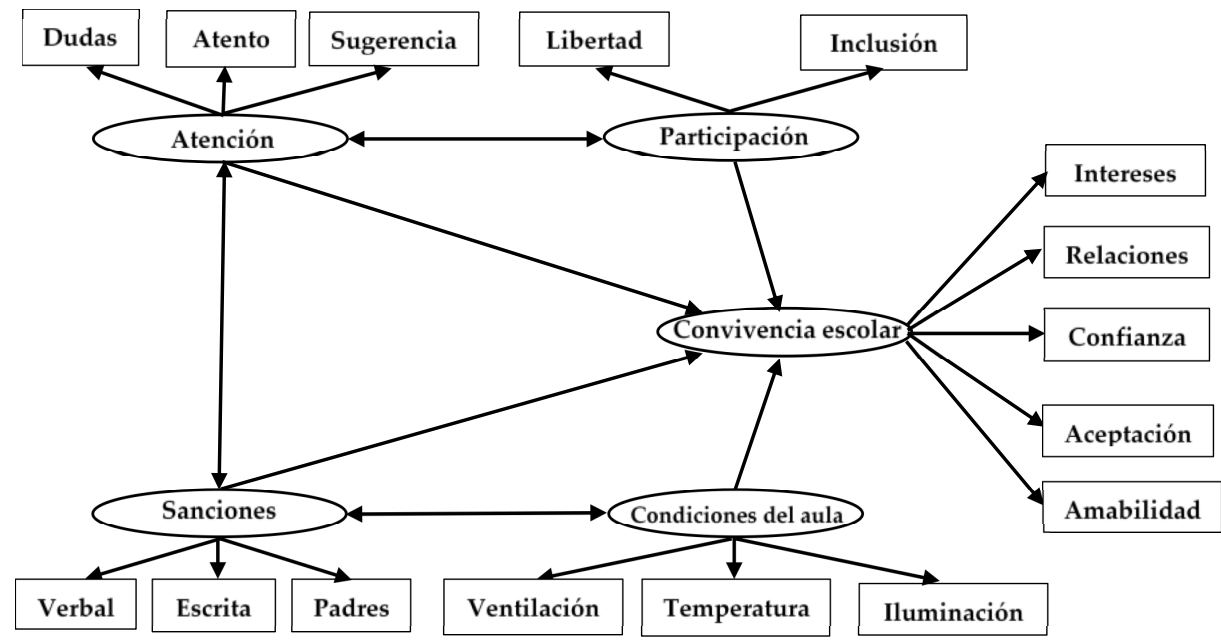

Figura 1. Modelo teórico

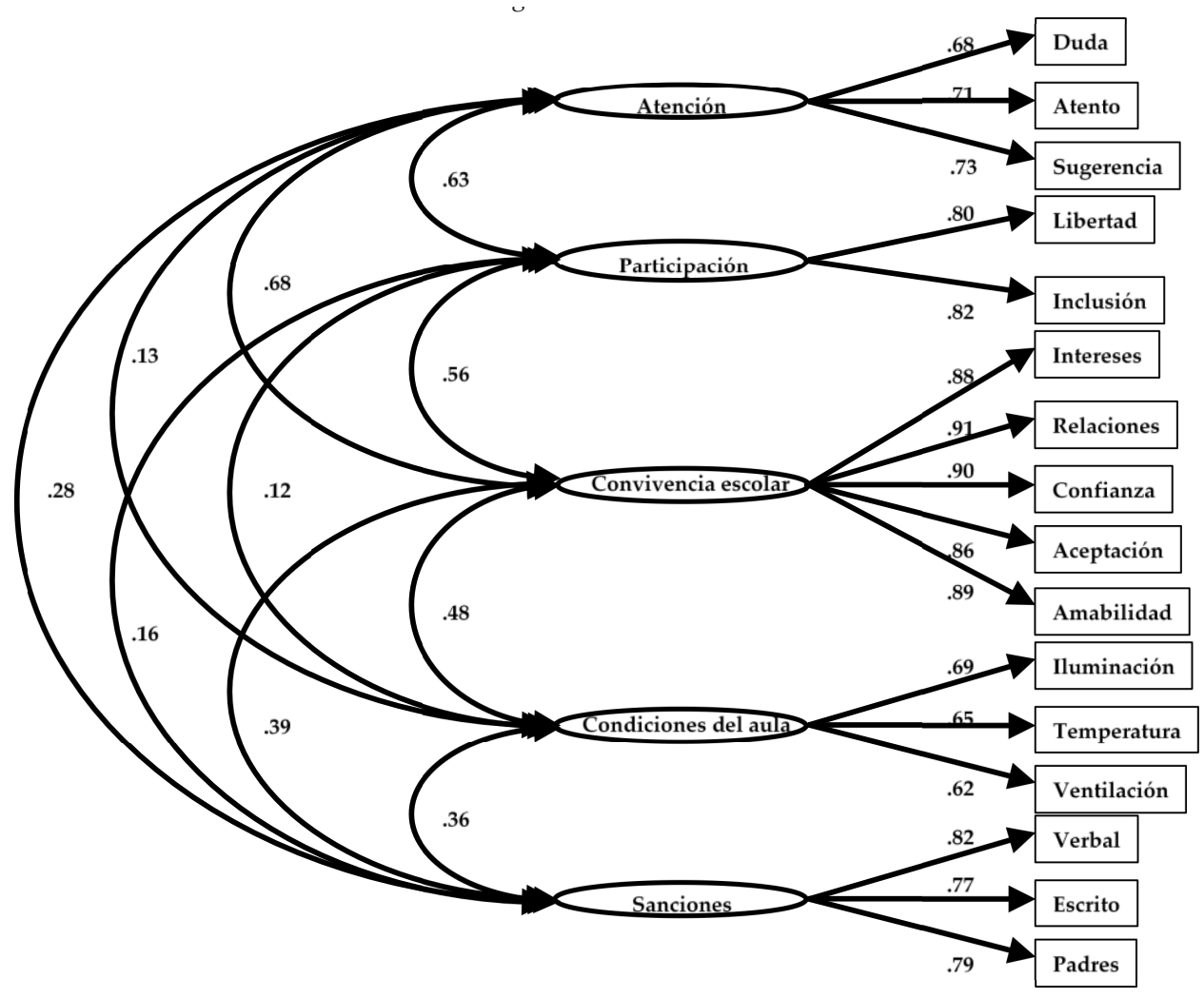

Figura 2. Análisis factorial confirmatorio 


\section{Procedimiento de recogida y análisis de datos}

La recogida de datos se llevó a cabo en octubre de 2016. Los estudiantes respondieron las preguntas del cuestionario en una sesión que duró 90 minutos en cada una de las escuelas.

Para realizar el análisis de datos y corroborar empíricamente el modelo teórico, fue aplicada la técnica de modelaje con ecuaciones estructurales. Debido a que las variables fueron de nivel ordinal, se empleó como método de estimación el de mínimos cuadrados ponderados, además, se obtuvieron el estadístico de bondad de ajuste Chicuadrado dividido por sus grados de libertad y algunos estadísticos de parsimonia e índices incrementales.

\section{Resultados}

Una vez aplicada la técnica de modelaje con ecuaciones estructurales, se obtuvo el modelo empírico (Figura 2). La Chi-cuadrado sobre sus grados de libertad $(\chi 2 / g . l$.) = 2.43 que debe ser menor a 3 para ser aceptado (Usluel, Aşkar \& Baş, 2008); el índice absoluto Error de Aproximación Cuadrático Medio (RMSEA) = .002 que tiene que ser menor a .05 (Ferrando \& Anguiano Carrasco, 2010) y el índice predictivo, que es el Índice de Validación Cruzada Esperada $(E C V I)=.975$ cuyo valor debe estar próximo a uno (Kline, 2005). Por otro lado, los índices incrementales o medidas de ajuste incremental fueron el Índice Normado de Ajuste $(\mathrm{NFI})=.961$, el cual debe ser de al menos .95 para ser aceptable (Ruiz, Pardo \& San Martín, 2010), el Índice de Tucker Lewis $(\mathrm{TLI})=.938$ con valores aprobados si el resultado como mínimo es 9 (Escobedo Portillo, Hernández Gómez, Estebané Ortega \& Martínez Moreno, 2016) y el Índice de Ajuste Normado de Parsimonia $(\mathrm{PNFI})=.982$ que debe ser aproximadamente igual a 1 (Lévy Mangin, 2003).

El factor que presentó un mayor peso estandarizado fue Atención (.41); el que los profesores aclaren dudas, estén atentos a los trabajos que realizan y hagan sugerencias sobre tareas y trabajos para que mejoren, se asocia con una mejora en la Convivencia escolar. El segundo factor que tuvo mayor peso estandarizado fue Participación (.28); cuando los profesores permiten que los alumnos participen y organicen sus clases de tal manera que sus estudiantes sientan la libertad de colaborar en ellas, la Convivencia escolar se ve favorecida.

El siguiente factor fue Condiciones del aula con un peso estandarizado de .17; mientras las aulas tengan una ventilación, temperatura e iluminación adecuadas, mejorará la Convivencia escolar. Por último, el factor Sanciones mostró un peso estandarizado de .08; al realizar sanciones de tipo amonestación verbal, escrita y citar a los padres de familia, se está contribuyendo a tener una mejor Convivencia escolar.

Por su parte, se encontró una correlación positiva y significativa entre el factor Atención con Participación y Sanciones de .78 y .09, respectivamente. Esto es, al incentivar la Participación en lo que respecta a la libertad e inclusión e implementar las sanciones verbales, escritas y citar a los padres, se contribuye a tener una mejor Atención. Además, Condiciones del aula se correlacionó positiva y significativamente (.11) con Sanciones. Dicho de otra manera, tener más aulas de clases con ventilación, 
temperatura e iluminación adecuadas se relaciona con una disminución en la cantidad de veces que se amonesta de forma verbal y escrita y en citar a los padres.

Con respecto a las variables medibles de mayor peso de regresión estandarizado en cada factor, se obtuvo que, cuando la Convivencia escolar aumenta en una desviación estándar, la amabilidad, aceptación y confianza crecen .70, .67 y .64 desviaciones estándar, respectivamente. Lo anterior significa que la Convivencia escolar mejora cuando los profesores son amables, aceptan la forma de ser de sus estudiantes y los escolares tienen confianza en sus maestros. Asimismo, por cada desviación estándar que se incremente las Condiciones del aula, la ventilación y temperatura aumentan .76 y .73 desviaciones estándar, respectivamente. Es decir, las Condiciones en el aula se ven favorecidas mientras que mejoren la ventilación y temperatura. Para el caso del factor Sanciones, por cada desviación estándar que este aumente, la amonestación escrita y el citar a los padres de familia crecen .87 y .62 desviaciones estándar, respectivamente.

Para la Atención, por cada desviación estándar que se incremente, las variables atentos y sugerencias crecen .64 y .60, respectivamente. En otras palabras, mientras los docentes estén atentos y hagan sugerencias hacia los alumnos, mejorará la Atención. Por último, por cada desviación estándar que aumente la Participación, la inclusión crece .67 desviaciones estándar. Esto es, cuando los profesores organizan sus clases para promover la inclusión y participación de los estudiantes en las actividades del aula, la Participación crece.

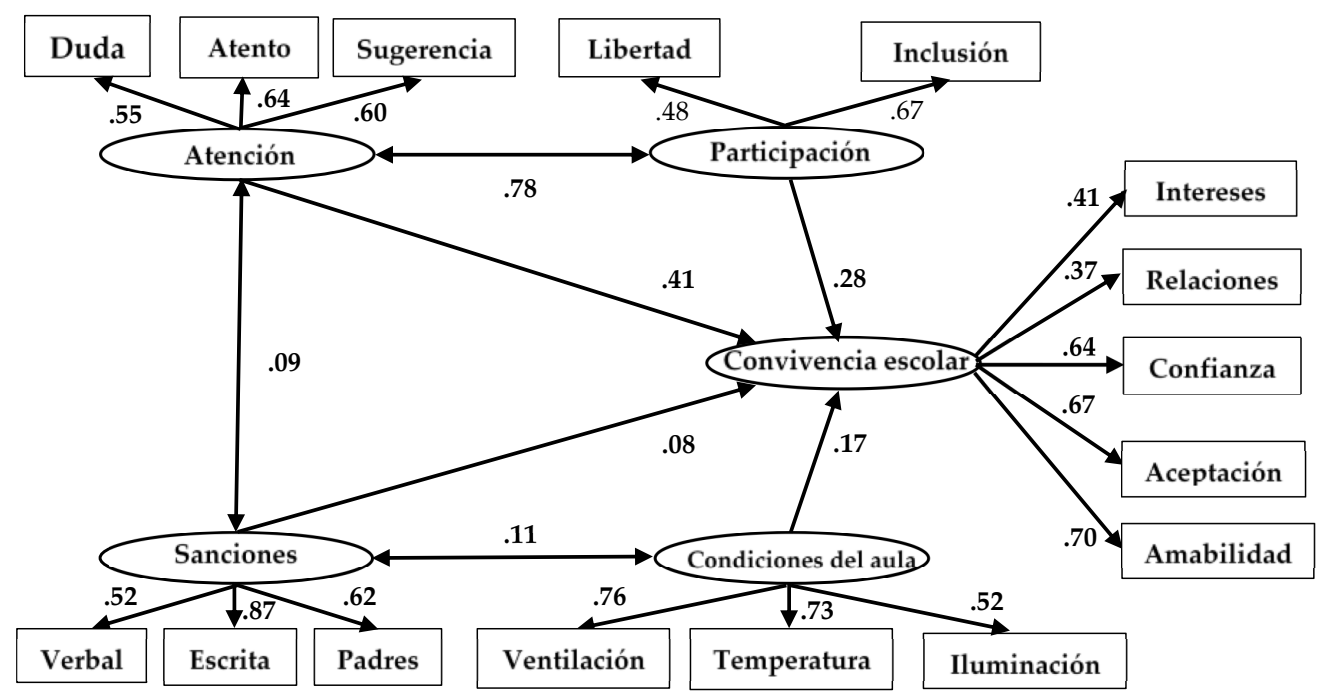

Figura 2. Modelo empírico 


\section{Discusión y Conclusiones}

En este trabajo se ha puesto en evidencia que, existe una relación entre los factores Atención, Participación, Sanciones y Condiciones del aula con la Convivencia escolar en estudiantes de bachillerato. A continuación, se discuten dichas relaciones.

De forma particular, la Atención que brindan los docentes a sus alumnos es crucial para mejorar la Convivencia escolar, tal como fue expresado desde la Secretaría de Educación del Gobierno del estado de México (2013) y por Arón et al. (2017). Dichas evidencias también coinciden con otros trabajos, en los cuales se abordaba que los maestros deberían orientar sus prácticas hacia la comunicación, el fomento del trabajo en equipo y el compromiso (Peña Figueroa et al., 2017), ser mediadores en los procesos de enseñanza y aprendizaje a través de sugerencias, razonamiento y persuasión (Parra, 2014), y favorecer la interacción con confianza y respeto (Castañeda Millán, 2014), esto con el fin de promover la convivencia, las relaciones positivas y los mejores ambientes.

Derivado de lo anterior, los maestros debieran: aclarar dudas durante las clases, mantenerse atentos en los ejercicios que se lleven a cabo y brindar consejos o sugerencias. Para ello, resulta relevante que el profesorado reflexione sobre los ajustes que tiene que hacer durante su labor (Artavia Granados, 2005) y que, además, reciba cierta capacitación para impulsar los ambientes de convivencia (Reyes Angona et al., 2018).

En el caso de la Participación se encontró que esta también favorece una sana Convivencia, información coincidente con Artavia Granados (2005), López de Mesa Melo et al. (2013), Muñoz Quezada et al. (2014), Red de Apoyo a la Gestión Educativa (2014) y Sandoval Manríquez (2014). Por tanto, los maestros debieran brindar a los alumnos la confianza para participar durante las clases e introducir ejercicios que favorezcan la participación de todos, así como se hizo saber desde el Departamento de Educación, Cultura y Deporte (2007), y por Escobar Medina (2015), García et al. (2006) y Murillo Pacheco (2010). Al respecto, este último autor señaló que dicho acto iría concibiendo aprendizajes como la tolerancia, el reconocimiento de los errores y la escucha asertiva. Asimismo, la idea de analizar el currículo escolar, con el propósito de que en este sobresalga la misma participación (Vidanes Díez, 2007) cobra importancia, siendo esta una forma de irla incorporando en el proyecto didáctico de las escuelas (Muñoz Quezada et al., 2014).

En lo que corresponde a las Sanciones (amonestación verbal, escrita y citar a los padres de familia), al ser aplicadas en la escuela, se está contribuyendo a alcanzar una mejor convivencia, un hallazgo coincidente hasta cierto punto con lo abordado desde la Secretaría de Educación Pública del estado de Puebla (2016), y por Ruiz Cuéllar (s.f.), Landeros y Chávez (2015) y Colom et al. (2010).

Cabría analizar la idea direccionada a la importancia que tienen los reglamentos escolares (en los cuales están incluidas las sanciones) para educar en la convivencia (Ochoa Cervantes \& Diez Martínez, 2013), siendo que dichas sanciones pudieran ser los exhortos por escrito y el entablar diálogos entre docentes, alumnos y padres (Gobierno del estado de Nuevo León, 2016). Además, los factores Atención, Participación y Sanciones se relacionan entre sí. Por ello, cuando se estimula la participación y se implementan las sanciones en los aspectos ya abordados, se está contribuyendo a contar con una mejor atención en el aula. 
Con respecto a las Condiciones del aula, mientras este espacio cuente con ventilación, temperatura e iluminación adecuadas, se estará mejorando la Convivencia escolar. Tal hallazgo coincide con otros reportes que han dado a conocer el valor de cumplir con estos criterios porque vienen a promover los procesos comunicativos (INIFED, 2013; SEP, s.f.a.) y el progreso armónico de las acciones (influyendo en las conductas del estudiantado) (Rodríguez Vite, s.f.), actos que están asociados con una adecuada convivencia.

Por otra parte, Condiciones del aula y Sanciones se relacionaron, razón por la cual es primordial que los salones de clases cuenten con una adecuada ventilación, temperatura e iluminación, una gestión que, de llevarse a cabo, podría estar disminuyendo la cantidad de amonestaciones verbales y escritas que reciben los alumnos y de citatorios para padres. Dicha manifestación ya había sido descrita de manera semejante por Morales Rivas et al. (2014); por ello, la propuesta de Quintero Corzo et al. (2015) cobra relevancia ya que va orientada a incluir en los planes de desarrollo, estrategias de mejora en las condiciones citadas para regular las interacciones en las escuelas.

En definitiva, se puede concluir que algunas acciones, tanto de los docentes (involucramiento) como de las autoridades educativas (condiciones del aula), pudieran dar paso a mejorar la convivencia escolar. Es un hecho que los maestros poseen en su actuar una herramienta muy valiosa. Por tanto, pequeñas modificaciones en sus labores estarían generando cambios positivos, de ahí la relevancia que tienen los docentes y todas las personas que de alguna u otra manera están involucradas en el mantenimiento y equipamiento de las aulas.

Finalmente, se debe resaltar que, tras el desarrollo del presente trabajo, se abren nuevas perspectivas para el abordaje de la convivencia escolar, esto es, la generación de un modelo teórico, examinado a través de una técnica multivariada avanzada y verificado empíricamente, brinda las pautas para realizar este tipo de análisis en investigaciones posteriores, un acto que permitirá establecer líneas claras de acción en la práctica educativa para beneficio de los alumnos de bachillerato.

\section{Referencias}

Alvarado Juárez, A. M. (2019). Convivencia escolar en el nivel medio superior: un estudio de caso en contexto de migración. Revista Iberoamericana de las Ciencias Sociales y Humanísticas, 8(15). doi: 10.23913/ricsh.v8i15.166

Arias Vinasco, I. C. (2018). Ambientes escolares: un espacio para el reconocimiento y respeto por la diversidad. Sophia, 14(2), 84-93. doi: 10.18634/sophiaj.14v.2i.852

Arón, A. M., Milicic, N., Sánchez, M., \& Subercaseaux, J. (2017). Construyendo juntos: claves para la convivencia escolar. Santiago de Chile, Chile: Agencia de Calidad de la Educación. Recuperado de http://archivos.agenciaeducacion.cl/Convivencia_escolar.pdf

Arredondo Reyes, D. (2019). Las prácticas pedagógicas y su incidencia en la convivencia escolar. Revista de Investigaciones UCM, 19(33), 64-76.

Alegría Rivas, L. M. E. (2016). Violencia escolar: las prácticas docentes y el abandono escolar. Ra Ximhai, 12(3), 397-413. 
Artavia Granados, J. M. (2005). Interacciones personales entre docentes y estudiantes en el proceso de enseñanza y aprendizaje. Revista Electrónica Actualidades Investigativas en Educación, 5(2), 1-19. Recuperado de https://www.redalyc.org/pdf/447/44750208.pdf

Barreda Gómez, M. S. (2012). El docente como gestor del clima del aula. Factores a tener en cuenta. (Tesis inédita de maestría). Universidad de Cantabria, Santander, España.

Bos, M. S., Ganimian, A. J., \& Vegas, E. (2014). Brief \# 9: ¿Cómo se relaciona el aprendizaje estudiantil con los recursos que se invierten en educación? Washington, D. C., Estados Unidos: Banco Interamericano de Desarrollo.

Caballero Grande, M. J. (2010). Convivencia escolar: un estudio sobre buenas prácticas. Revista Paz y Conflictos, 3, 154-169.

Cámere, E. (2009). La relación profesor-alumno en el aula. Entre Educadores. Educación, Familia \& Valores. Recuperado de https://entreeducadores.com/2009/08/01/la-relacionprofesor-alumno-en-el-aula/

Castañeda Millán, F. J. (2014). Hacia una interacción constructiva: una propuesta de alumnos de preparatoria. Revista Iberoamericana para la Investigación y el Desarrollo Educativo, 5(9), 42-55.

Castro Pérez, M., \& Morales Ramírez, M. E. (2015). Los ambientes de aula que promueven el aprendizaje, desde la perspectiva de los niños y niñas escolares. Revista Electrónica Educare, 19(3), 1-32. doi: 10.15359/ree.19-3.11

Centro de Estudio y Acción Social Panameño (2012). Estudio de patrones de convivencia escolar en Panamá. Ciudad de Panamá, Panamá: Centro de Estudio y Acción Social Panameño. Recuperado de https://www.unicef.org/panama/spanish/convivenciaescolar_2013.pdf

Colom, A. J., Castillejo, J. L., Pérez Alonso, P. M., Rodríguez, T., Sarramona, J., Touriñán, J. M., \& Vázquez, G. (2010). Violencia, convivencia y educación: claves para la intervención pedagógica en la escuela. Revista de Investigación en Educación, 8, 6-23.

Contreras, S. I. (2018). Convivencia escolar y solución de conflictos mediante la investigación como estrategia pedagógica. Cultura, Educación y Sociedad, 9(3), 63-72. doi: 10.17981/cultedusoc.9.3.2018.07

Cruz Hernández, M. Z., \& Reyes Ruiz, M. T. (2016). Convivencia escolar y RIEMS: un diagnóstico de la séptima competencia docente. Ra Ximhai, 12(3), 323-333.

Chavez Romo, C., Gómez Nashiky, A., Ochoa Cervantes, A., \& Zurita Rivera, Ú. (2016). La política nacional de convivencia escolar de México y su impacto en la vida en las escuelas de educación básica. Revista Posgrado y Sociedad, 14(1), 1-13.

Departamento de Educación, Cultura y Deporte (2007). Convivencia en los centros educativos. Zaragoza, España: Departamento de Educación, Cultura y Deporte. Recuperado de https://convivencia.files.wordpress.com/2008/10/cuento_contigo_mod4-209p1.pdf

Díaz Better, S. P., \& Sime Poma, L. E. (2016). Convivencia escolar: una revisión de estudios de la educación básica en Latinoamérica. Revista Virtual Universidad Católica del Norte, 49, 125-145.

Dopico Rodríguez, E. (2011). Conflicto y convivencia en los entornos escolares. Cuadernos de Educación y Desarrollo, 3(26). Recuperado de http://www.eumed.net/rev/ced/26/ edr.htm

Escobar Medina, M. B. (2015). Influencia de la interacción alumno-docente en el proceso enseñanza-aprendizaje. Paakat: Revista de Tecnología y Sociedad, 8. 
Escobedo Portillo, M. T., Hernández Gómez, J. A., Estebané Ortega, V., \& Martínez Moreno, G. (2016). Modelos de ecuaciones estructurales: características, fases, construcción, aplicación y resultados. Ciencia E Trabajo, 18(55), 16-22.

Ferrando, P. J., \& Anguiano Carrasco, C. (2010). El análisis factorial como técnica de investigación en psicología. Papeles del Psicólogo, 31(1), 18-33.

Fierro Evans, C., \& Carbajal Padilla, P. (2019). Convivencia escolar: una revisión del concepto. Psicoperspectivas, 18(1). doi: 10.5027/psicoperspectivas-vol18-issue1-fulltext-1486

García, M., Olvera, C., \& Flores, J. (2006). Vínculo de comunicación alumno-maestro en el aula. Razón y Palabra, 54. Recuperado de http://www.razonypalabra.org.mx/ anteriores/n54/vinculos.html

García Cabrero, B., Loredo Enríquez, J., \& Carranza Peña, G. (2008). Análisis de la práctica educativa de los docentes: pensamiento, interacción y reflexión. Revista Electrónica de Investigación Educativa, Especial, 1-15. Recuperado de https://redie.uabc.mx/redie/ article/view/200

Gobierno del estado de Nuevo León (2016). Reglamento de disciplina escolar de las escuelas de educación públicas y particulares de educación básica y media superior del estado de Nuevo León. Monterrey: Gobierno del estado de Nuevo León. Recuperado de http://www. nl.gob.mx/sites/default/files/reglamento_de_disciplina_escolar.pdf

González Medina, M. A., \& Treviño Villarreal, D. C. (2018). Logro educativo y factores asociados en estudiantes de sexto grado de educación primaria en el estado de Nuevo León, México. Perfiles Educativos, 40(159), 107-125.

Hernández Prados, M. A., \& Sánchez Romero, E. (s.f.). La convivencia escolar, una tarea pendiente en los padres. Santiago de Chile, Chile: Educrea. Recuperado de https://educrea.cl/la-convivencia-escolar-una-tarea-pendiente-en-los-padres/

Ianni, N. D. (2003). La convivencia escolar: una tarea necesaria, posible y compleja. Monografías Virtuales. Ciudadanía, democracia y valores en sociedades plurales, 2. Recuperado de https://www.oei.es/historico/valores2/monografias/monografia02/reflexion02.htm

Instituto Nacional de la Infraestructura Física Educativa (2013). Diseño arquitectónico. Educación básica-secundaria. México: Instituto Nacional de la Infraestructura Física Educativa. Recuperado de http://www.inifed.gob.mx/doc/normateca/tec/CR/03_CDASEC-GRAL-TEC-TELES.pdf

Instituto Nacional para la Evaluación de la Educación (2016a). Evaluación de condiciones básicas para la enseñanza y el aprendizaje desde la perspectiva de los derechos humanos. México: Instituto Nacional para la Evaluación de la Educación. Recuperado de https:// publicaciones.inee.edu.mx/buscadorPub/P1/E/201/P1E201.pdf

Instituto Nacional para la Evaluación de la Educación (2016b). Infraestructura, mobiliario y materiales de apoyo educativo en las escuelas primarias. ECEA 2014. México: Instituto Nacional para la Evaluación de la Educación. Recuperado de https://publicaciones. inee.edu.mx/buscadorPub/P1/D/244/P1D244.pdf

Instituto Nacional para la Evaluación de la Educación (2018). La convivencia escolar en las escuelas primarias de México. México: Instituto Nacional para la Evaluación de la Educación. Recuperado de https://publicaciones.inee.edu.mx/buscadorPub/P1/D/250/ P1D250.pdf

Kline, R. B. (2005). Principles and practice of structural equation modeling. Nueva York, Estados Unidos: Guilford Press. doi: 10.1177/1049731509336986 
Landeros, L., \& Chávez, C. (2015). Convivencia y disciplina en la escuela. Análisis de reglamentos escolares de México. México: Instituto Nacional para la Evaluación de la Educación. Recuperado de https://publicaciones.inee.edu.mx/buscadorPub/P1/C/232/P1C232.pdf Lévy Mangin, J. P. (2003). Modelización y análisis con ecuaciones estructurales. Madrid, España: Prentice Hall.

López, V. (2014). Convivencia escolar. Apuntes, 4, 1-18. Recuperado de http://www.unesco. org/new/fileadmin/MULTIMEDIA/FIELD/Santiago/pdf/APUNTE04-ESP.pdf

López de Mesa Melo, C., Carvajal Castillo, C. A., Soto Godoy, M. F., \& Urrea Roa, P. N. (2013). Factores asociados a la convivencia escolar en adolescentes. Educación y Educadores, 16(3), 383-410

Luna Rivera, E. (2015). La importancia de establecer espacios libres de violencia y conflicto en los contextos educativos. México: Universidad Mexicana.

Ministerio de Educación (s.f.). Convivencia escolar. Santiago de Chile, Chile: Ministerio de Educación. Recuperado de http://www.convivenciaescolar.cl/index2.php?id_ seccion=3375\&id_portal=50\&id_contenido=13803\#clima

Miranda López, F. (2018). Infraestructura escolar en México: brechas traslapadas, esfuerzos y límites de la política pública. Perfiles Educativos, 40(161), 32-52.

Morales Rivas, C. P., Marina Narváez, E., \& Sarria Membreño, A. E. (2014). Implementación de acciones metodológicas dirigidas a disminuir la indisciplina escolar de los estudiantes de noveno grado del colegio Apostólico El Lirio de los Valles de la ciudad de León en el segundo semestre del año lectivo 2013. León: Universidad Nacional Autónoma de Nicaragua-León.

Muñoz Quezada, M. T., Lucero Moncada, B. A., Cornejo Araya, C. A., Muñoz Molina, P. A., \& Araya Sarabia, N. E. (2014). Convivencia y clima escolar en una comunidad educativa inclusiva de la Provincia de Talca, Chile. Revista Electrónica de Investigación Educativa, 16(2), 16-32.

Murillo Pacheco, H. (2010). Misión del docente: propiciar en el estudiante aprendizajes significativos. Enfermería Universitaria, 7(4), 42-52.

Ochoa Cervantes, A., \& Diez Martínez, E. (2013). El reglamento escolar como eje de análisis de la convivencia en la escuela. Ensaio: Avaliação e Políticas Públicas em Educação, 21(81), 667-684.

Oficina Regional de Educación para América Latina y el Caribe (2017). Suficiencia, equidad y efectividad de la infraestructura escolar en América Latina según el TERCE. Santiago de Chile, Chile: Oficina Regional de Educación para América Latina y el Caribe.

Parra, K. N. (2014). El docente y el uso de la mediación en los procesos de enseñanza y aprendizaje. Revista de Investigación, 38(83), 155-180.

Penalva López, A. (2018). La convivencia escolar. Un reto del siglo XXI. Revista Electrónica de Investigación y Docencia, 20, 41-58. doi: 10.17561/reid.n20.3

Peña Figueroa, P. P., Sánchez Prada, J., Ramírez Sánchez, J., \& Menjura Escobar, M. I. (2017). La convivencia en la escuela. Entre el deber ser y la realidad. Revista Latinoamericana de Estudios Educativos, 13(1), 129-152.

Pérez Archundia, E., \& Gutiérrez Méndez, D. (2016). El conflicto en las instituciones escolares. Ra Ximhai, 12(3), 163-180.

Pérez Galván, L. M., \& Ochoa Cervantes, A. C. (2017). La participación de los estudiantes en una escuela secundaria: retos y posibilidades para la formación ciudadana. 
Revista Mexicana de Investigación Educativa, 22(72). Recuperado de http://www.scielo. org.mx/scielo.php?script=sci_arttext\&pid=S1405-66662017000100179

Pineda Alfonso, J. A., \& García Pérez, F. F. (2016). Conflicto y convivencia: profesores y alumnos en el proceso de enseñanza en un aula de secundaria. Revista Mexicana de Investigación Educativa, 21(71), 1073-1091.

Quintero Corzo, J., Munévar Molina, R. A., \& Munévar Quintero, F. I. (2015). Ambientes escolares saludables. Revista de Salud Pública, 17(2), 229-241. doi: 10.15446/rsap. v17n2.35882

Red de Apoyo a la Gestión Educativa (2014). La convivencia en los centros educativos de educación básica en Iberoamérica. Santiago de Chile, Chile: Red de Apoyo a la Gestión Educativa. Recuperado de https://dd.uab.cat/pub/llibres/2014/131430/ Red_AGE_2014_web.pdf

Reyes Angona, S., Gudiño, S., \& Fernández Cárdenas, J. M. (2018). Violencia escolar en Michoacán y Nuevo León, un diagnóstico situado para promover escuelas seguras en educación básica. Revista Electrónica de Investigación Educativa, 20(2), 46-58. doi: 10.24320/redie.2018.20.2.1548

Rodríguez Vite, H. (s.f.). Ambientes de aprendizaje. México: Universidad Autónoma del Estado de Hidalgo. Recuperado de https://www.uaeh.edu.mx/scige/boletin/huejutla/ n4/e1.html

Ruiz, M. A., Pardo, A., \& San Martín, R. (2010). Modelos de ecuaciones estructurales. Papeles del Psicólogo, 31(1), 34-45.

Ruiz Cuéllar, G. (s.f.). Violencia y disciplina en escuelas primarias y secundarias. México: Instituto Nacional para la Evaluación de la Educación. Recuperado de http://publicaciones.inee.edu.mx/buscadorPub/P1/B/204/P1B204_09C04.pdf

Sandoval Manríquez, M. (2014). Convivencia y clima escolar: claves de la gestión del conocimiento. Última Década, 41, 153-178.

Secretaría de Educación del Gobierno del estado de México (2013). Manual para docentes mexiquenses. Aprender a convivir en una cultura de paz. Programa de valores por una convivencia escolar armónica de la Secretaría de Educación del Gobierno del Estado de México. México: Secretaría de Educación del Gobierno del Estado de México. Recuperado de https://fondodeculturaeconomica.com/PDF/Caja\%20de\%20herramientas/Manual\%20 para\%20docentes\%20mexiquenses.\%20Aprender\%20a\%20vivir\%20en\%20paz.pdf

Secretaría de Educación Pública (s.f.a). Espacios educativos y aprendizaje. Orientaciones para fortalecer la gestión escolar. México: Secretaría de Educación Pública. Recuperado de https://www2.sepdf.gob.mx/petc/archivos-documentos-rectores/espacios_educativos_aprendizaje.pdf

Secretaría de Educación Pública (s.f.b). ¿Qué es la atención? México: Secretaría de Educación Pública. Recuperado de http://www.construye-t.org.mx/resources/pdf/ lecciones/leccion4/4.4_E_Que_es_la_atencion_CExperimentales.pdf

Secretaría de Educación Pública (2008). Glosario. Términos utilizados en la dirección general de planeación y programación. México: Secretaría de Educación Pública. Recuperado de http://cumplimientopef.sep.gob.mx/2010/Glosario\%202008\%2024-jun-08.pdf

Secretaría de Educación Pública (2016). Manual para el docente. Educación primaria del programa nacional de convivencia escolar. México: Secretaría de Educación Pública. 
Recuperado de https:/www.gob.mx/cms/uploads/attachment/file/147157/PNCEMANUAL-DOC-PRIM-BAJA.pdf

Secretaría de Educación Pública del estado de Puebla (2016). Manual para el mejoramiento de la convivencia escolar a partir de la reflexión sobre la práctica docente. Puebla: Secretaría de Educación Pública del estado de Puebla. Recuperado de http://www. itssmt.edu.mx:2020/SGC/descargar2.php?id=2143

Usluel, Y. K., Aşkar, P., \& Baş, T. (2008). A Structural Equation Model for ICT Usage in Higher Education. Educational Technology \& Society, 11(2), 262-273.

Vidanes Díez, J. (2007). La educación para la paz y la no violencia. Revista Iberoamericana de Educación, 42. Recuperado de https://rieoei.org/historico/deloslectores/ experiencias146.htm

Fecha de recepción: 15 de abril de 2019.

Fecha de revisión: 25 de julio de 2019.

Fecha de aceptación: 24 de noviembre de 2019. 